\title{
Immune response to tissue stress
}

Immune activation occurs not only in response to infection, but also following physical, chemical and genotoxic tissue stress. Two recent studies by Hayday and colleagues reveal new aspects in stress-initiated immune responses (termed lymphoid stress-surveillance responses) in humans and mice.

Natural killer group 2, member D (NKG2D) is expressed by natural killer (NK) cells, $\gamma \delta$ T cells and some $\mathrm{CD}^{+} \alpha \beta$ T cells, and its ligation induces cytotoxic activity and cytokine expression. In human tissues, sterile stress results in the upregulation of the NKG2D ligand MICA (MHC class I polypeptide-related sequence $\mathrm{A}$ ). In an attempt to characterize the role of highly polymorphic MICA in human lymphoid stresssurveillance responses, Shafi et al. introduced a single copy of different MICA alleles into an identical genomic site in Chinese hamster ovary epithelial cells. Subsequent

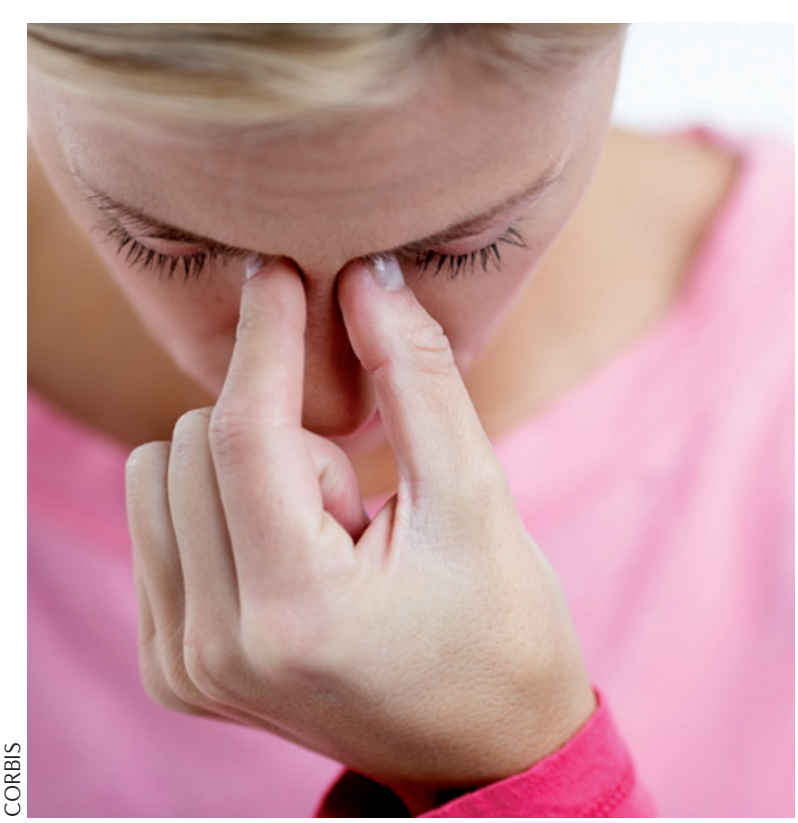

analysis showed that intragenic variation resulted in different RNA and protein expression levels of each MICA allele.

The cell-surface expression of different MICA alleles (the levels of which largely reflected the allelespecific differences in total protein expression) resulted in the killing of MICA-transfected cells by human peripheral blood mononuclear cells (PBMCs), and this involved both NK cells and $\gamma \delta$ T cells, but not $\mathrm{CD}^{+} \alpha \beta$ T cells. However, PBMCs from different donors had varying responses to the different MICA alleles; some donors responded optimally to highly expressed MICA alleles and others to lower MICA expression. Thus, the authors suggest that the differential levels of basal expression of MICA alleles in each individual (as determined by the individual's MICA haplotype, as well as other genetic and environmental factors) tune $\mathrm{NKG}_{2} \mathrm{D}^{+}$ lymphocytes to respond to changes of MICA expression in a hostspecific manner. This would ensure optimum responsiveness only to the levels of MICA upregulation that are associated with tissue stress in the individual, while maintaining tolerance to the host-specific homeostatic levels of MICA.

In a complementary study, Strid et al. investigated how epidermal $\gamma \delta$ $\mathrm{T}$ cells respond to the upregulation of the mouse MICA homologue, RAE1, following cutaneous epithelial stress. Mild epidermal abrasion was found to induce a rapid but transient upregulation of RAE1 expression, and this was accompanied by a transient induction of cytokine expression in the skin. The induction of the T helper 2 $\left(\mathrm{T}_{\mathrm{H}}\right.$ 2)-type cytokines interleukin-4 (IL-4) and IL-13 depended on the presence of $\gamma \delta$ T cells, indicating that epidermal $\gamma \delta$ T cells that are activated by mild cutaneous tissue stress might favour the initiation of $\mathrm{T}_{\mathrm{H}}$ 2-type immune responses.

Interestingly, when cutaneous epithelial stress was combined with the epicutaneous application of ovalbumin, high levels of ovalbuminspecific IgG, IgG1 and IgE, as well as of total IgE, were measured in the blood. Whereas IgG1 production was dependent on $\alpha \beta$ T cells, IgE production (which is often associated with atopic allergies) largely depended on the presence of epidermal $\gamma \delta$ T cells. Secondary exposure to ovalbumin induced even higher levels of $\gamma \delta$ $\mathrm{T}$ cell-dependent IgE production, suggesting that $\gamma \delta$ T cells that are initially activated in the skin by mild stress can support long-term systemic $\mathrm{T}_{\mathrm{H}}$ 2-type immune responses. Finally, studies in mice deficient either for NKG2D (which mediates lymphoid stress surveillance) or for MYD88 (a molecule associated with Toll-like receptor signalling that mediates responses to microorganisms) showed that a substantial component of IL-13 and IgE production depended on NKG2D but not on microbial stimulation. Hence, the authors propose that the increasing incidence of atopic allergies might reflect not only altered exposure to environmental microorganisms but also frequent exposure to environmental toxins that induce sterile tissue stress.

Maria Papatriantafyllou

ORIGINAL RESEARCH PAPERS Shafi, S. et al. An NKG2D-mediated human lymphoid stresssurveillance response with high interindividual variation. Sci. Transl. Med. 30 Nov 2011 (doi:10.1126/science.3002922) |Strid, J. et al. The intraepithelial T cell response to NKG2Dligands links lymphoid stress surveillance to atopy Science 334, 1293-1297 (2011) 